\title{
A SUBMILLIMETER MISSION FOR THE 1990s: SMMM
}

\author{
T.G. PHILLIPS \\ Downes Laboratory, California Institute of Technology, Pasadena, CA
}

\section{Introduction}

Submillimeter wavelengths hold the key to some of the most important aspects of astronomy. These range from star-forming molecular clouds and proto-planetary disks in our galaxy to infrared emitting galaxies at cosmological distances. Indeed, the essential problems of star-formation and galaxy-formation will be directly probed by the submillimeter spectral lines and continuum radiation emitted by these objects. Other fascinating topics falling into the submillimeter band include the Wien component of the cosmic background radiation, containing information on the nature of the early universe, and nearer to home, the spectroscopy of planetary atmospheres. Since the submillimeter contains fundamental information on the physics and chemistry of so many aspects of our universe, every effort should be made to provide the very best instrumentation for these astronomical studies. We should be capable of detection and analysis of even the most distant objects yet conceived.

Telescopes specifically designed for submillimeter astronomy are now operating on high mountain sites and the field is developing in an exciting and rapid fashion. NASA's airborne program has been in operation for some time and has been of the greatest importance in getting the field started. Both ground and airborne programs will continue to be essential because of their flexibility for implementing new investigations, for instrument development and to support the growth of an active science community, especially students. However, it is now essential to move forward on a space program.

\section{The Need to be above the Atmosphere}

Space telescopes will be qualitatively and quantitatively superior to any groundbased or suborbital telescope for at least three reasons:

- The Earth's atmosphere blocks the spectral coverage of ground-based telescopes, except for wavelengths longward of $350 \mu \mathrm{m}$. Even at the altitudes of airborne telescopes, at least half of the submillimeter spectrum $(100 \mu \mathrm{m}$ to $1 \mathrm{~mm})$ is blocked or confused.

- Emission by the Earth's atmosphere and from the relatively warm telescopes limits the sensitivity of both ground-based and airborne telescopes.

- Observing time is extremely limited with balloon or airborne telescopes and is subject to strict weather requirements for ground-based telescopes operating at the highest frequencies.

Figure 1 shows the transmission of the atmosphere from: a) the 45,000-foot altit ude of the KAO or future airborne telescopes; b) a high mountain, such as Mauna Kea, Hawaii. Apart from balloons, low water vapor ground sites and airplanes 

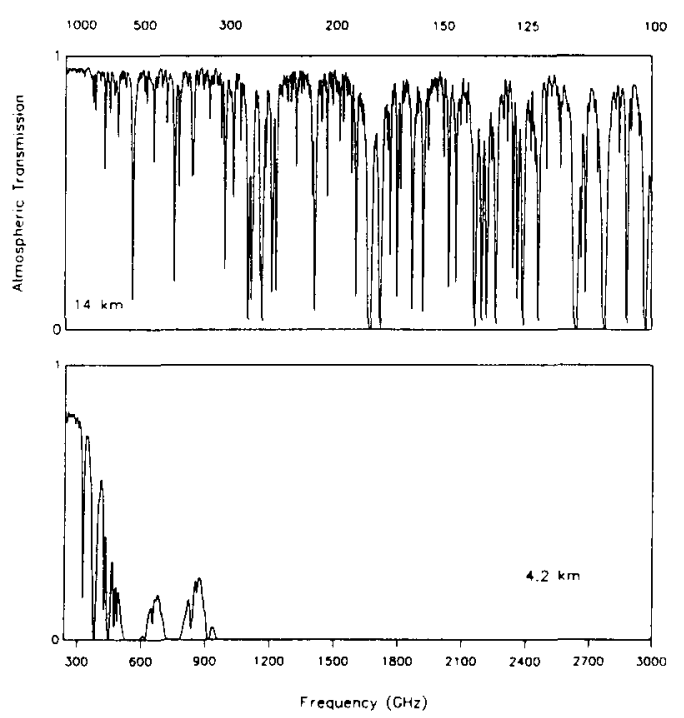

Fig. 1. Transmission of the atmosphere from: a) 45,000-ft KAO altitude and b) a 14,000-ft mountain site.

represent the only likely non-space platforms for observing in the submillimeter range. It is clear from Figure 1 that mountain-based telescopes can only be used in a few selected, longer wavelength windows.

The South Pole site, while an improvement on Hawaii atmospherically, only allows a restricted range of southern observations and does not approach the transmission quality obtained by the KAO. The atmospheric spectrum from airborne altitudes is complex and depends upon the resolution of the detection instrumentation. Although certain spectral lines (e.g., those of C II, C I, O I) can be observed quite clearly through the airborne atmosphere, particularly with high-resolution Fabry-Perot or heterodyne techniques, it is not possible to make detailed observations of many species, including the critically important molecules $\mathrm{H}_{2} \mathrm{O}$ and $\mathrm{O}_{2}$.

Furthermore, it is not possible to carry out a spectral survey or to obtain a broad overview of the combined spectral output from dust (which may have as yet unobserved resonances in the submillimeter band), molecules, and atoms. Finally, even those specific lines which may be well observed within the Galaxy would not necessarily be observable for red-shifted galaxies.

\section{The Submillimeter Mission}

This report describes a 'Moderate Mission' spacecraft concept, designed to make physical and chemical studies of high red-shift galaxies, molecular clouds, starforming regions and planets. SMMM will enormously enhance our knowledge of 


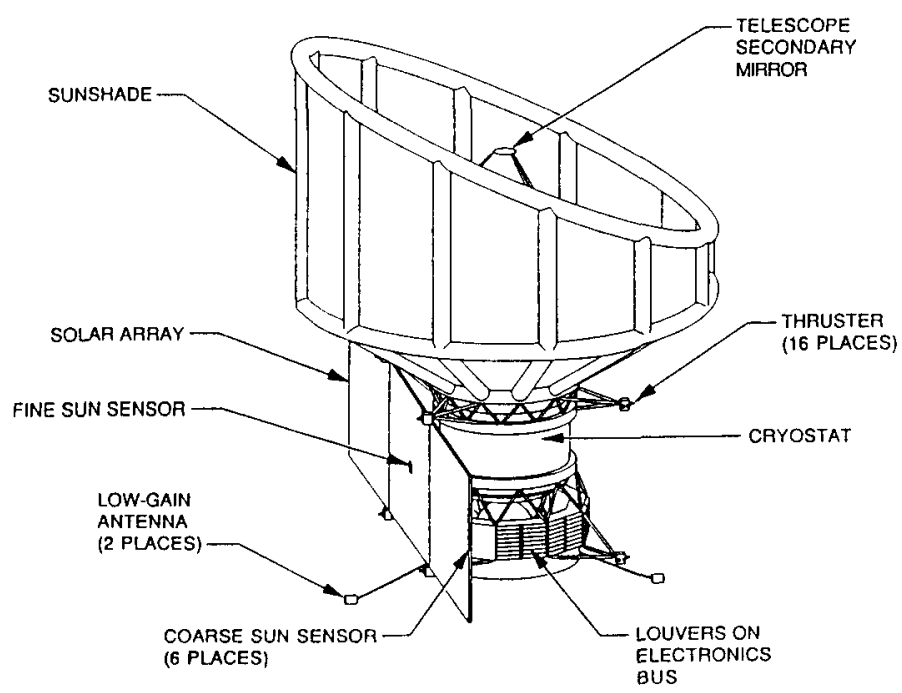

Fig. 2. SMMM spacecraft flight configuration

these objects and provide a pioneering spectroscopic survey of the submillimeter band. It will also have excellent capabilities for cosmological studies in the continuum. At the same time it will generate essential experience for both astronomers and technologists in this new and difficult field and test many of the concepts needed for 'Major Observatory' class missions, such as LDR and the possible Lunar Interferometer, which cannot themselves be achieved in the 1990s time frame.

SMMM (Figure 2) will utilize a roughly $4 \mathrm{~m}$ ambient telescope with a liquidhelium cooled focal plane. It will be in a high elliptical orbit $(1 \mathrm{k} \times 70 \mathrm{k} \mathrm{km})$ which will provide an excellent observing environment. It will achieve a complete submillimeter spectroscopic survey of a large number of sources between 100 and $700 \mu \mathrm{m}$. Spectroscopy is to be carried out by heterodyne techniques at the long wavelengths and by direct detection techniques in conjunction with a far-infrared spectrometer at shorter wavelengths. In addition to the spectroscopy, SMMM will have a far-infrared camera with a bolometer array. The expected lifetime is about 2 years.

Very great advances in submillimeter instrumentation have been made in recent years. Heterodyne receivers now use superconducting tunnel junction detectors (SIS) which are extremely sensitive and approach $10 \%$ quantum efficiency. Although relatively commonplace in the optical, this is very hard to achieve at radio or submillimeter wavelengths. Very recently major strides have been made in bolometers cooled to $0.1 \mathrm{~K}$, so that NEP's of $10^{-17} \mathrm{~W} / \sqrt{\mathrm{Hz}}$ can be obtained which will allow SMMM to approach background limited operation, even in the case of the high resolution Fabry-Perot spectrometer. For typical resolutions of $10^{4}$ the line sensitivity will be better than $10^{-18} \mathrm{~W} \mathrm{~m}^{-2}$ in 1000 seconds. In the continuum $0.3 \mathrm{~K}$ bolometers will achieve point source sensitivities of about $1.5 \mathrm{mJy}$ in 1000 seconds. For the telescope, new carbon epoxy materials are available for panel manufacture and new techniques of construction will provide sufficient accuracy to easily reach diffraction limited performance at $100 \mu \mathrm{m}$. The components for submillimeter astronomy in 
space are approaching readiness.

This mission represents the central 1990's component of a phased NASA plan for the submillimeter region. The overall plan, starting with the Small Scout class Explorer (SWAS), leading to SMMM and finally (although not in the 1990s) to LDR and a possible Lunar Interferometer, is described in a separate "Workshop Report on Submillimeter Planning" submitted separately. SMMM benefits from the initial science investigation and component qualification performed by the $.5 \mathrm{~m}$ Scout class mission, SWAS, which is already approved.

The current status of SMMM is that it is undergoing pre-phase A study by NASA, jointly with the French Space Agency CNES. Since the programmatic situation is uncertain, it is being studied as either a 'Moderate Mission' with a 3.7 $\mathrm{m}$ aperture, to be launched by an Atlas rocket, or as an 'Explorer' with a $2.5 \mathrm{~m}$ aperture, to be launched by a Delta. Recently CNES approved the funding for its portion of the phase A study for SMMM.

For the following discussion it is assumed that the $3.7 \mathrm{~m}$ version will be constructed.

\section{The Submillimeter Astronomy Goals}

The submillimeter spectral range contains the clues to processes by which diffuse, dusty gas with temperatures of 10 to $100 \mathrm{~K}$ is assembled into stars and planetary systems, both within the Galaxy and in other galaxies. At such temperatures, the peak emission occurs in the submillimeter band, approximately $100 \mu \mathrm{m}$ to $1 \mathrm{~mm}$. Unlike visible radiation, which scarcely penetrates even diffuse gas, submillimeter radiation can probe across the galaxy and into the cores of even the densest condensations.

Most galaxies and sources in star formation regions, discovered in the Infrared Astronomical Satellite (IRAS) survey, have their greatest flux longward of $100 \mu \mathrm{m}$. Very distant and possibly primeval galaxies emit radiation in the mid-infrared, due to dust and atomic fine-structure lines, which will be red-shifted into the submillimeter. The IRAS survey, supplemented by surveys of tracers of molecular gas such as $\mathrm{CO}$, has identified the major nearby sources of far-infrared and submillimeter radiation; however, the resolution of IRAS was limited to 1 to 3 arc minutes in four very broad wavelength bands, shortward of $100 \mu \mathrm{m}$. To understand the detailed physics of far-infrared sources, what is needed now is accurate high spatial and spectral resolution measurements of specific objects. A $4 \mathrm{~m}$ class telescope will give angular resolutions of between 6 and 18 arc seconds in the $100-300 \mu \mathrm{m}$ range. Heterodyne and Fabry-Perot techniques will give spectral resolutions up to $3 \times 10^{6}(0.1 \mathrm{~km} / \mathrm{sec})$ and $10^{4}(30 \mathrm{~km} / \mathrm{sec})$ respectively. These capabilities are ideal for studies of interstellar gas in the Galaxy and distant galaxies.

Atomic and molecular submillimeter spectra of interstellar gas contain information on a scale rivalling that of optical spectra. They are immensely useful and versatile diagnostics of physical conditions, such as temperature, density, and velocity structure. Because of their rich vibrational-rotational spectra, molecules can probe regions ranging from cold quiescent clouds to hot star-forming regions. In addition, they play an important active role in the energy balance of these ob- 
jects by providing the major coolants. The amount of energy released by molecular rotational and atomic fine-structure emission at millimeter and submillimeter wavelengths determines, in part, the rates of collapse and, ultimately, the efficiency of star formation in molecular clouds. The amount of energy released is also very sensitive to the exact chemical composition of the gas and dust. Equally, molecular and atomic ions are of great importance, because they determine the coupling of the gas to the magnetic field.

Molecules and molecular ions can also be used as chemical diagnostics of important astrophysical parameters, such as the cosmic-ray ionizing frequency and the overall deuterium abundance and its gradient across the Galaxy. The abundances of some species may be particularly enhanced in star-forming regions, so that these molecules could be used as signposts of the initial stages of the star-formation process. Microwave masers are a traditional example of this. Another application would be the use of molecules as "clocks" to determine ages of molecular clouds and, thus, shed light on the evolution of such clouds. Good progress has been made in understanding aspects of gas-phase chemistry, but the role of grain-surface chemistry, the exchanges between grain-mantles and the gas-phase, and the link between large molecules and very small grains is not understood. This is exactly the area in which SMMM will be of most use, because it would provide us with a complete view of chemical abundances in a large diversity of regions through broadband spectroscopic capabilities, as well as understanding of the dust-grain behavior in the same region, through spectro-photometric and high-angular resolution capabilities.

The major science goals for SMMM fall into two categories: 1) a complete submillimeter line-survey, and 2) galaxies and cosmology. The submillimeter line-survey applies in the main to observations of galactic clouds which require high resolution spectroscopy for analysis of their physical and chemical properties.

\subsection{A Complete Submillimeter Line-Survey}

The ability to obtain a complete submillimeter spectrum for approximately 150 sources, in a two year lifetime, will provide a major step forward in fields ranging from star-formation and interstellar medium chemistry and physics to planetary atmospheres. Some important aspects are now discussed.

\subsubsection{An unbiased overview of atomic and molecular abundances in gas clouds of various types throughout the galaxy}

Figure 3 shows what we anticipate will be the submillimeter spectrum of a typical molecular cloud at a temperature of $30 \mathrm{~K}$. It is a composite of the spectrum of dust, heavy and light molecules and ions and fine structure transitions of atoms and atomic ions. The amazing complexity of heavy molecule rotational spectra at long wavelengths will merge, in the submillimeter, into the fundamental rotation spectra of light molecules, typified by metal hydrides.

Figure 4 indicates the quality of spectrum obtainable with SIS receivers on the ground, through an atmospheric window at $1.3 \mathrm{~mm}$. This quality will be extended through the submillimeter band in the regime of the SIS receivers and, because of 


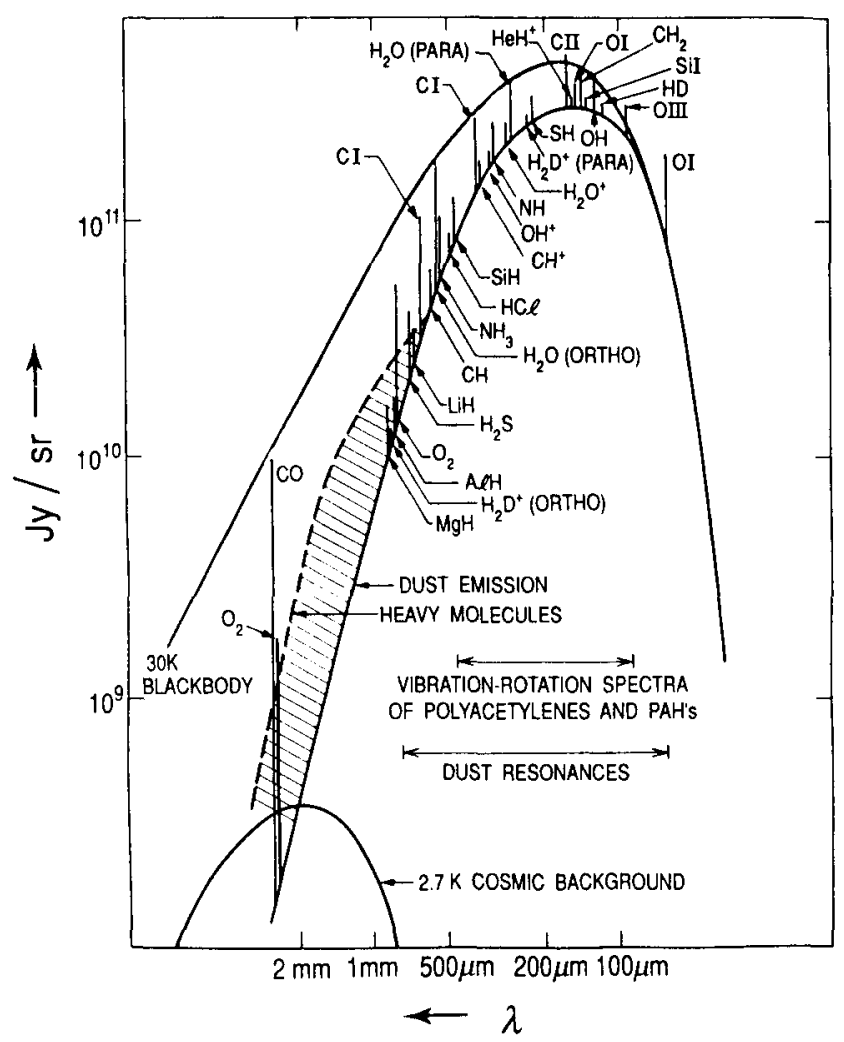

Fig. 3. The anticipated spectrum of a $30 \mathrm{~K}$, dense interstellar cloud, showing the dust spectrum, heavy and light molecule rotation spectra and atomic fine-structure lines

the very high sensitivity of the new bolometers, will be approximately matched by the $10^{4}$ resolution Fabry-Perot spectrometer.

The major advantages of a line-survey, aside from the obvious one of completeness are the certainty of identification due to the multiple lines for each species and the ability to solve the radiation transfer and molecule excitation problems, again because of the multiline advantage and also because of the consistent and more accurate calibration. These factors, lead to far better values for abundances than are available from single line studies. Equally important are the possibilities for discoveries!

\subsubsection{Thermal Balance and Dense Regions}

The heating of the interstellar medium involves both microscopic processes (e.g. absorption of ultraviolet starlight by atoms and molecules) and macroscopic phenomena (e.g. heating by shock waves and cloud-cloud collisions). The heating is bal- 

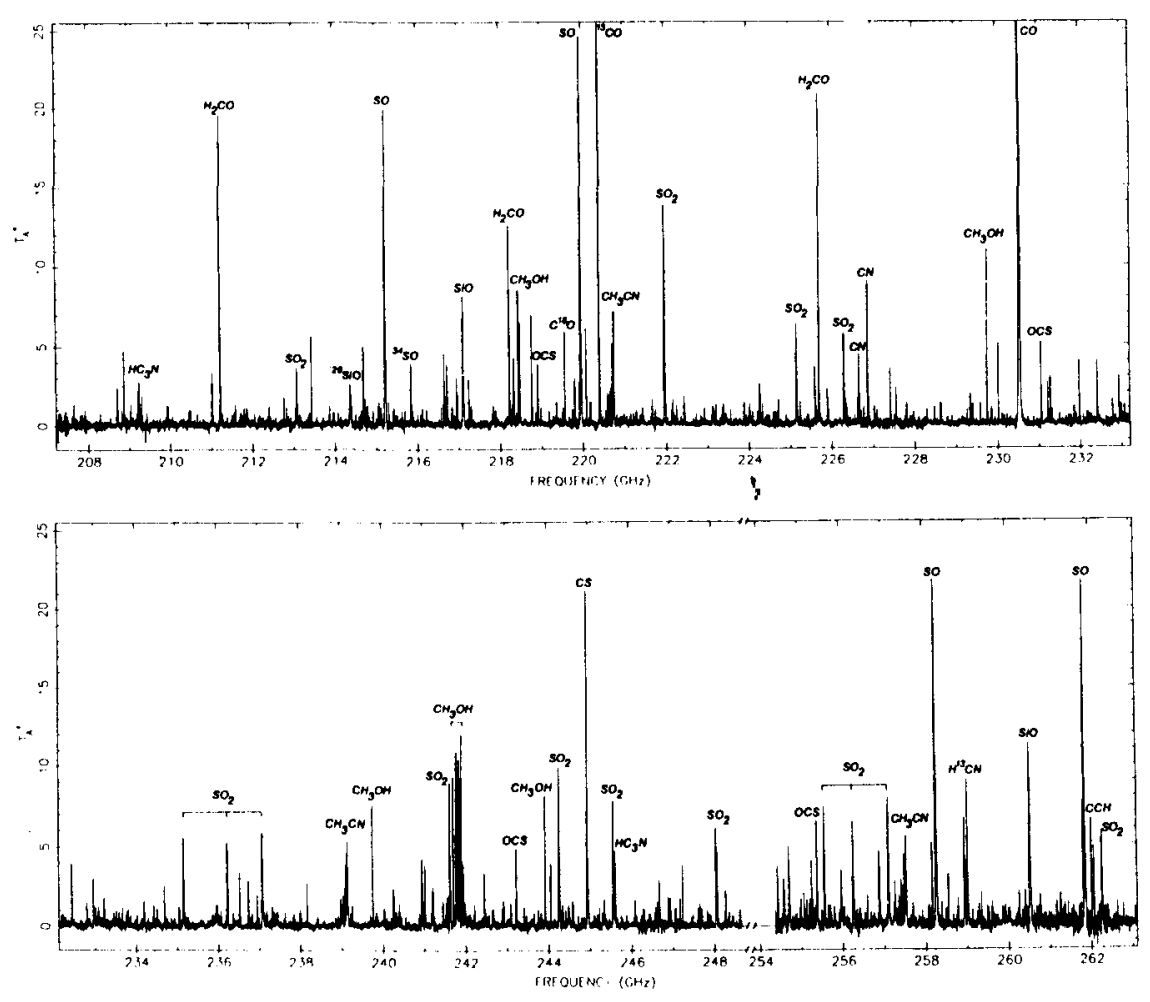

Fig. 4. A spectrum of the core of the Orion Molecular Cloud, taken in the $1.3 \mathrm{~mm}$ window using SIS receivers. This shows the richness and quality of spectrum obtainable with sensitive receivers, when the atmospheric absorption is negligible

anced by the radiation emitted by the interstellar gas and dust. The structure of the interstellar medium, i.e., how its pressure responds to gravitational and magnetic forces, is regulated by this thermal balance. Most of the neutral interstellar medium is characterized by temperatures $T \approx 10$ to $100 \mathrm{~K}$, and the bulk of its thermal radiation thus lies in the submillimeter region of the spectrum. Observation of the submillimeter radiation from the gas and dust of interstellar clouds can thus be used to assess their internal and external energy sources and to probe the processes that shape them and turn some of them into stars.

The gas and dust components can behave independently in many interstellar clouds, even though the gas and dust are believed to be well mixed. In the interstellar medium of our Galaxy, the dust exchanges approximately 100 times as much radiant energy with its surroundings as the gas; however, the dust and gas are in poor thermal contact with each other at densities below $10^{6} \mathrm{~cm}^{-3}$. The dust radiates continuously with a few broad resonances in the infrared, while the gas radiates through a myriad of discrete, narrow lines. In some warm molecular clouds, 
notably OMC-1, the lines are known to contribute a significant fraction of the broad band continuum flux at $\lambda \approx 1 \mathrm{~mm}$ and presumably at shorter wavelengths also. A particular advantage of SMMM is that it can survey completely much of the line cooling spectrum of an interstellar cloud simultaneously with the thermal emission of the dust component. A critical point in the collapse of a cloud core to form a star must occur when the dust first becomes opaque at the wavelengths $(\lambda \approx 1$ to $0.1 \mathrm{~mm}$ ) of maximum emission at $T \approx 10$ to $100 \mathrm{~K}$. At subsequent stages of the collapse, virtually none of the cooling radiation escapes freely from the cloud center and the dust and gas components become strongly coupled through radiation. Submillimeter observations can thus be used to infer the evolutionary status of star-forming clouds.

Owing to its rich submillimeter spectrum and its expected high abundance, $\mathrm{H}_{2} \mathrm{O}$ is predicted to be an extremely important coolant, not only in cold clouds, but also in hotter, shock-heated regions. In dense clouds, the rotational excitation of $\mathrm{H}_{2} \mathrm{O}$ will be controlled by a complicated combination of collisional processes, radiative transfer, and radiative coupling to the dust. Only with the full spectral coverage of SMMM will it be possible to assess thoroughly the excitation of this important coolant and its relation to cloud structure. Figure 5 displays the cooling rates per $\mathrm{H}_{2}$ of typical interstellar molecular gas and indicates the dominance of $\mathrm{H}_{2} \mathrm{O}$ and other light hydrides at high densities.

The critical density at which a submillimeter transition is observed rapidly increases with frequency. Figure 6 shows the conditions of excitation required for a number of atomic and molecular species. SMMM will be able to study lines throughout the density-temperature space of the figure and will allow the assessment of the thermal balance of clouds over a very wide density range.

\subsubsection{Star-Formation regions}

The understanding of star-formation is directly related to the determination of the structure of interstellar gas at various scale sizes. The formation and evolution of low-mass stars can be studied by spectral surveys of a few nearby clouds in the Taurus and Ophiuchus regions, by examining density fluctuations in quiescent clouds, and by mapping the distribution of continuum radiation at high spatial resolution. The question of fossil protostellar disks near main sequence stars can also be addressed.

SMMM can make important spectroscopic measurements toward low-mass protostars, such as HL Tau, L1551, and IRAS 16293-2422. Such systems are probably similar to the pre-solar nebula and observations of them will provide insight into the physical and chemical structure of our own primitive solar system. Virtually all theories about the conditions in the pre-solar nebula are based upon very indirect evidence from meteorites, comets, and planetary atmospheres.

The prevailing view is that in formation of the pre-solar nebula, matter from the placental molecular cloud falls onto an accreting disk and passes through a shock front, where the densities increase from $10^{4}$ to $10^{6} \mathrm{~cm}^{-3}$ in the precursor cloud, to $10^{8}$ to $10^{12} \mathrm{~cm}^{-3}$ in the disk. In the inner regions, the temperatures are high $(>1000 \mathrm{~K})$ and all the dust is evaporated. As the disk cools, the atoms and 


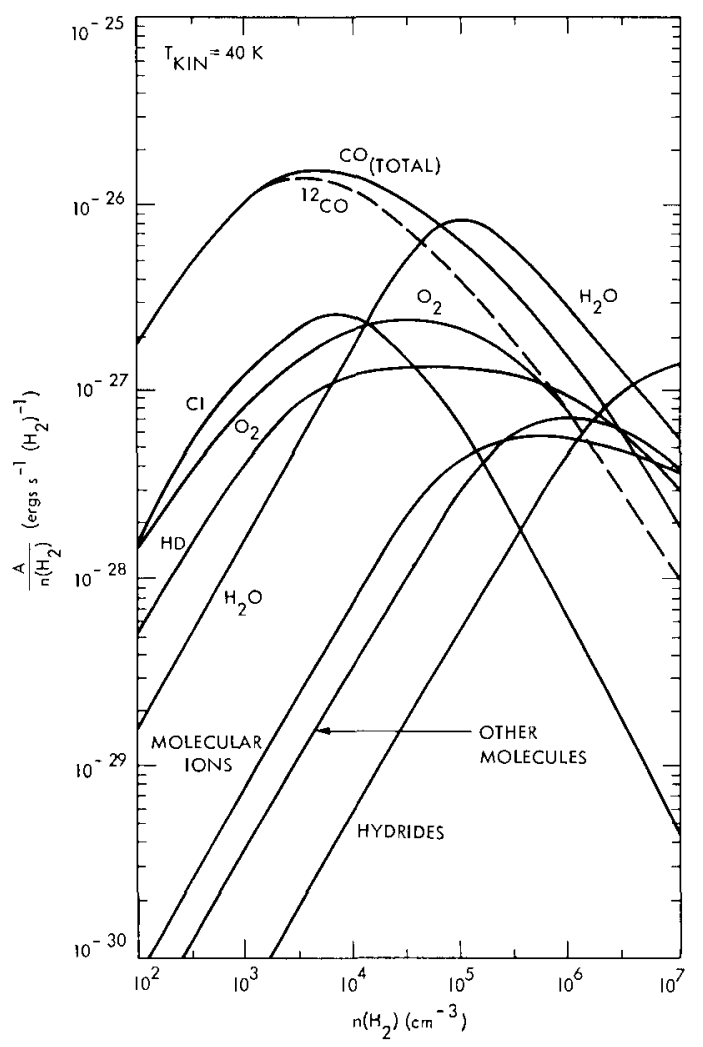

Fig. 5. Cooling rates per $\mathrm{H}_{2}$ molecule, showing the dominance of $\mathrm{H}_{2} \mathrm{O}$ and hydrides at the high densities of star-forming regions

molecules are thought to condense in sequence according to their abundance, local vapor pressure, and ambient temperature, resulting in a very different chemical mix from that of the parent interstellar cloud. SMMM will be able to study the innermost disks surrounding nearby low-mass protostars through the strengths of abundant species and through the velocity distribution as given by emitted spectral line profiles, ev en though these regions are too tiny $\left(\leq 1^{\prime \prime}\right)$ to map directly. Highlevel transitions of molecules such as $\mathrm{H}_{2} \mathrm{O}, \mathrm{CS}$, and $\mathrm{HCN}$, which trace high-density regions, will be particularly prominent from the hot, dense inner regions.

A study of the chemical structure of the outer region of such disks is also of interest to cometary studies. Theories of comet formation put their origins anywhere between 30 and 10,000 AU. These objects are, therefore, thought to contain the most primitive solar nebula material, which has undergone the least processing in the inner nebula. However, the inferred abundances for Comet Halley indicate $\mathrm{H}_{2} \mathrm{O}$ is by far the most abundant species. The observations of the chemical abundances in circumstellar disks may reveal whether the chemical environment during comet formation is different from that in the dense clouds. If not, this would provide 


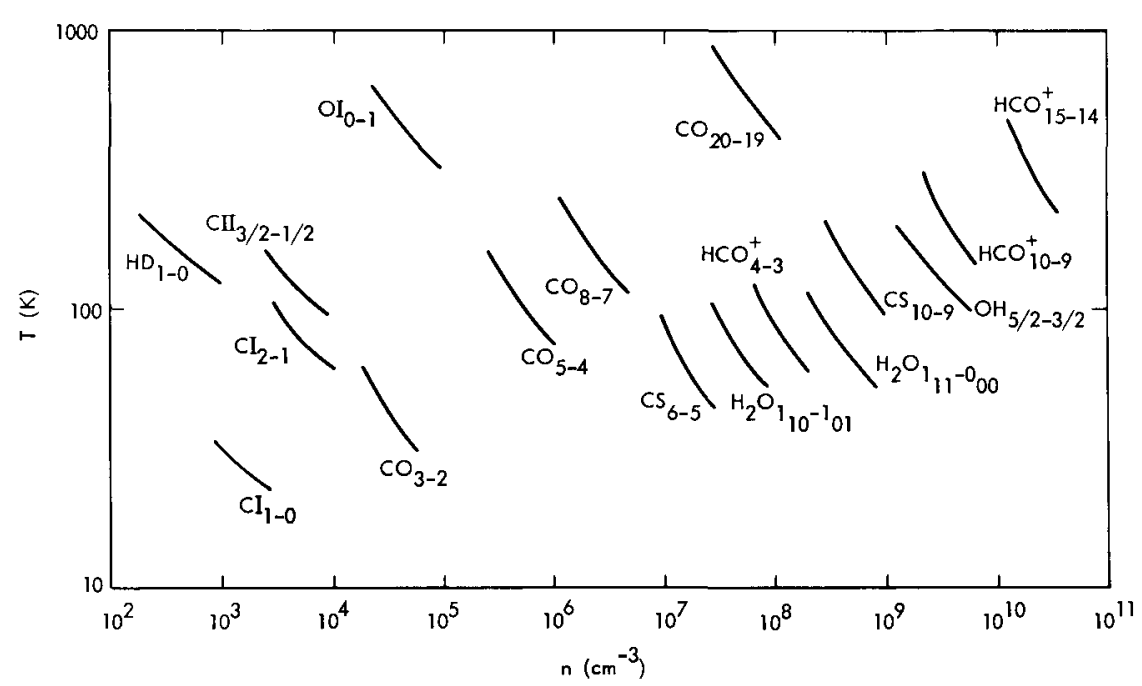

Fig. 6. Indicates the very wide density-temperature range available to SMMM

a strong indication that comets undergo subsequent processing in the inner solar nebula.

The critical molecule for these observations is $\mathrm{H}_{2} \mathrm{O}$, which cannot be observed from the ground. For the strongest lines of $\mathrm{H}_{2} \mathrm{O}$ and for an assumed abundance of $10^{-6}$ relative to $\mathrm{H}_{2}$, SMMM will provide line profiles for these regions in only minutes. From the structure in the line shapes the mass and temperature distributions may be deduced.

The SMMM continuum camera will be extrememly useful for studies of protoplanetary disks. One of the most exciting results of the IRAS mission was the discovery that approximately one-third of all main sequence stars of types A through $K$ have far-infrared excesses attributable to solid material in stable orbits around the stars. Infrared or visual observations of the brightest of these stars show that this material is located in disks 10 to $500 \mathrm{AU}$ in size, very similar in scale to those seen around younger, but more distant pre-main-sequence stars. If disks are an integral part of the star formation process, then the disks surrounding stars like Beta Pictoris and Alpha Lyrae (Vega) are presumably remnants of the original disks, and their study is of direct relevance to the questions of star and planetary formation.

The sensitivity and spatial resolution of the SMMM camera are well suited to the study of these objects. While SIRTF and ISO have the sensitivity to study these sources, by finding them at distances as large as a $\mathrm{kpc}$ or searching for dust spectral features, the spatial resolution of these small cryogenic telescopes is limited, longward of $100 \mu \mathrm{m}$, where these sources emit strongly and radiative transfer effects are minimized. In particular, high spatial resolution maps at $100 \mu \mathrm{m}$ with $6^{\prime \prime}$ resolution (50 to $100 \mathrm{AU}$ at typical distances of 8 to $16 \mathrm{pc}$ ) will be critical for understanding the temperature and density structure of the disks around the nearest stars. For example, the presence of gaps or enhancements in the radial density 
distribution may be signposts to the formation of planets. Further, long wavelength information is critical for determining the size of the emitting grains and the total mass of the disk material. SMMM will have excellent sensitivity for studying the Vega phenomenon.

For example, Vega, which has a disk of average brightness, emits $7 \mathrm{Jy}$ at 100 $\mu \mathrm{m}$ from a region about $25^{\prime \prime}(200 \mathrm{AU})$ in diameter. If this emission were spread out uniformly, the surface brightness would result in $0.4 \mathrm{Jy}$ per $6^{\prime \prime}$ beam of SMMM, detectable in a few seconds of integration. The optical occultation observations of Beta Pictoris reveal emission extending over an arcminute (800 AU). Simple models show that SMMM will be able to follow the infrared emission out at least as far as the presently known optical emission. It will, thus, be possible to make a detailed comparison of the optical and infrared emissions, which is important for determining the distribution and physical properties of dust throughout the disk.

\subsubsection{Hydride molecules}

The principal rotational transitions of light hydride molecules lie in the submillimeter wavelength region. Many of these transitions coincide with strong atmospheric features, so that observations even from an airplane are not feasible, except in a few specific cases. The hydrides are often the most important molecules both for understanding chemical synthesis and, as already mentioned, for determining physical processes such as cooling. Here we just give a few examples.

One of the most important hydrides that SMMM will detect is $\mathrm{H}_{2} \mathrm{D}^{+}$. In the now highly favored ion-molecule theory much of the gas-phase chemistry in dense clouds is thought to be driven by reactions of neutrals with the $\mathrm{H}_{3}^{+}$ion. However, since $\mathrm{H}_{3}^{+}$has no strongly allowed rotational transitions, it has so far eluded discovery. Measurements of the $\mathrm{H}_{2} \mathrm{D}^{+}$lines would provide direct information on the $\mathrm{H}_{3}^{+}$ abundance in dense clouds, which, in turn, would put direct limits on the cosmicray ionizing frequencies to which the clouds are exposed. Also, most of the observed remarkable deuterium fractionation in heavy molecules, such as DCN and $\mathrm{DCO}^{+}$, is thought to derive from reactions with $\mathrm{H}_{2} \mathrm{D}^{+}$.

The broad frequency coverage of SMMM is especially important for the study of hydrides with identical nuclei, like $\mathrm{H}_{2} \mathrm{O}, \mathrm{H}_{2} \mathrm{D}^{+}, \mathrm{H}_{3} \mathrm{O}^{+}$, and $\mathrm{CH}_{2}$, for which the fundamental transitions of their semi-independent ortho- and para-species lie at quite different frequencies. Measurements of both species are needed for determining total abundances. Moreover, the relative populations of ortho- and para-species forms may represent a very interesting interplay among processes of formation, destruction, and excitation.

Hydrides whose abundances are of cosmological significance are $\mathrm{HD}$ and $\mathrm{LiH}$. The HD $J 0=(1 \rightarrow 0) 112-\mu \mathrm{m}$ line is hard to detect from an airplane due to its low dipole moment, which makes it difficult to distinguish from the dust background and interference from the atmosphere. However, the line will be readily observable with SMMM: Assuming a cosmic deuterium abundance of HD, it can be shown that the intensity relative to the continuum would be $4 \%$ at a spectral resolution of $10^{4}$. Observations of HD throughout the Galaxy could provide information on possible gradients with galactic radius. $\mathrm{LiH}$ is expected to be significant in the early uni- 
verse, but primordial LiH may or may not have survived in the interstellar medium. However, in regions exposed to intense cosmic-ray fluxes, such as clouds near the galactic center, the original ${ }^{6} \mathrm{Li} /{ }^{7} \mathrm{Li}$ abundance ratio may be significantly enhanced by spallation reactions, which could be probed through observations of the corresponding hydride ratio. LiH should also be detectable in red-giant mass-loss shells where the abundance is higher than usual. Observations across the Galaxy may provide new information on star-formation and nucleosynthesis rates as a function of galactic radius. Such studies can be compared with abundance determinations from visible spectroscopy. Very different information will be obtained this way because submillimeter spectroscopy can probe dense, obscured star-formation regions and galactic nuclei.

In general submillimeter line-survey spectroscopy will tell us the abundances of hydride species over much of the periodic table. It is probably the only way to discover exotic species such as $\mathrm{HeH}^{+}$. However, overall the most important hydride molecule is $\mathrm{H}_{2} \mathrm{O}$, for its role in oxygen chemistry and in cloud cooling.

\subsubsection{Dominant Carbon and Oxygen Species}

The carbon to oxygen ratio in the interstellar gas is probably the most important quantity in determining the chemical constitution of the molecular and atomic gas in dense regions and the resulting physical properties such as cooling. At the moment it is not even certain whether oxygen or carbon is more abundant.

The principal carbon- and oxygen-bearing species in interstellar clouds are usually predicted to be $\mathrm{CO}, \mathrm{H}_{2} \mathrm{O}$, and $\mathrm{O}_{2}$. Of these, only the column density of $\mathrm{CO}$ is reasonably well determined observationally. Little is known about the abundances of $\mathrm{H}_{2} \mathrm{O}$ and $\mathrm{O}_{2}$, especially in cold dark clouds.

KAO observations of atomic C I and C II lines in a limited set of objects have shown that these species are widespread throughout molecular clouds, a result which is both unexpected and important in the context of fundamental physical and chemical processes. It is anticipated that future high-angular resolution, groundbased observations of $\mathrm{C}$ I from submillimeter telescopes combined with low-angular resolution measurements from SWAS, will provide more insight into the atomic carbon chemistry. Future observations of the C II $158-\mu \mathrm{m}$ line (and its isotopic variety ${ }^{13} \mathrm{C}$ II) will be possible from space platforms such as ISO and SIRTF, but at a rather coarse angular resolution of only 1.6 '. The C II beam size of about $10^{\prime}$ obtainable with SMMM is much better matched to the $\mathrm{C} I$ beams provided by the ground-based telescopes; especially for the study of star-forming regions, such high angular resolution will be crucial.

Oxygen may be largely in the form of $\mathrm{H}_{2} \mathrm{O}$, the most important and probably the most abundant molecule to be searched for with SMMM. Reliable information about its abundance in interstellar clouds can only be obtained from space-based survey instruments, owing to the severe interference from telluric $\mathrm{H}_{2} \mathrm{O}$ features even at 14 $\mathrm{km}$ and the need to observe several lines of any species. $\mathrm{H}_{2} \mathrm{O}$ is, of course, known to be present in the interstellar medium from its famous maser-transition $\left(6_{16}-5_{23}\right)$ in the ortho branch at $22 \mathrm{GHz}$, but this line involves highly excited states $(>500 \mathrm{~K}$ above ground state) and provides little information about the general abundance of 
water. SWAS will be able to detect a single ortho- $\mathrm{H}_{2} \mathrm{O}$ line $\left(1_{10}-1_{01}\right.$ at $\left.557 \mathrm{GHz}\right)$ in a number of warm, dense sources, but that mission will lack the sensitivity and angular resolution to observe $\mathrm{H}_{2} \mathrm{O}$ more generally in colder molecular clouds. Since most $\mathrm{H}_{2} \mathrm{O}$-containing regions are likely to be small in extent, the eight times higher angular resolution of SMMM will be crucial. The overall improvement in sensitivity on that one line will be about two orders of magnitude. In fact SMMM would be able to detect the $557 \mathrm{GHz}$ fundamental ortho-line in dark cloud cores, in 1,000 seconds, up to a distance of $2 \mathrm{kpc}$ even if the abundance of $\mathrm{H}_{2} \mathrm{O}$ is at the low end of current estimates $\left(10^{-7}\right.$ relative to $\left.\mathrm{H}_{2}\right)$. It could easily detect giant cloud cores throughout the Galaxy. For the fundamental para-line at 1,100 $\mathrm{GHz}$ giant cloud cores could be detected to about $10 \mathrm{kpc}$, again on the most pessimistic abundance assumption. Moreover, because of its broad wavelength coverage, SMMM will be able to detect many $\mathrm{H}_{2} \mathrm{O}$ lines of both ortho- and para-modifications, which will provide accurate abundance determinations. At the same time, these lines will provide interesting information about the $\mathrm{H}_{2} \mathrm{O}$ formation and excitation (which is strongly affected by far-infrared pumping) and will, thus, put constraints on the physical conditions in the clouds. Observations of several $\mathrm{H}_{2} \mathrm{O}$ lines, simultaneously, within a small beam will also provide insight into the vexing problem of the maser excitation of this molecule in interstellar clouds, and maybe even in external galaxies where "megamasers" are observed.

Molecular oxygen, $\mathrm{O}_{2}$, is predicted by models to be a major reservoir of oxygen in dense interstellar clouds. However, its observation is also complicated by the presence of numerous terrestrial $\mathrm{O}_{2}$ features and it has not yet been detected. SWAS will be able to search for the ${ }^{16} \mathrm{O}_{2}$ line at $487 \mathrm{GHz}$, but may have only marginal detection capability. As for $\mathrm{H}_{2} \mathrm{O}$, SMMM will have the advantage of two orders of magnitude higher sensitivity, higher angular resolution, and multiline capabilities, which will allow detection of many additional $\mathrm{O}_{2}$ lines. Since the $\mathrm{O}_{2}$ transitions are only magnetic dipole allowed, the levels are easily populated even in regions of relatively low density, so that, from this point of view, the molecule should be detectable throughout the interstellar medium.

Chemical models predict that a low $\mathrm{H}_{2} \mathrm{O}$ abundance is coupled to a high $\mathrm{O}_{2}$ abundance, or vice versa. This conjecture can be tested directly by SMMM, since it will be able to provide accurate abundances for both species.

\subsubsection{Vibration - Rotation Spectroscopy of Small Grains and Heavy Molecules}

The spectral range of SMMM is ideally suited for studying the transition region between large, heavy molecules and dust-grains. This poorly understood part of ISM physics and chemistry has recently received considerable attention because of the discovery that small grains consisting of $\sim 50$ atoms can be transiently heated by the absorption of single photons to radiate strongly throughout the infrared. The possible identification of these small grains with large, planar graphite-like molecules called polycyclic aromatic hydrocarbons suggests that the apparent dearth of interstellar particles between dust-grains $0.1 \mu \mathrm{m}$ in size and molecules containing about 13 atoms could be due to the difficulty in observing large molecules which rotate too slowly to be found by millimeterwave spectroscopy, rather than a basic 


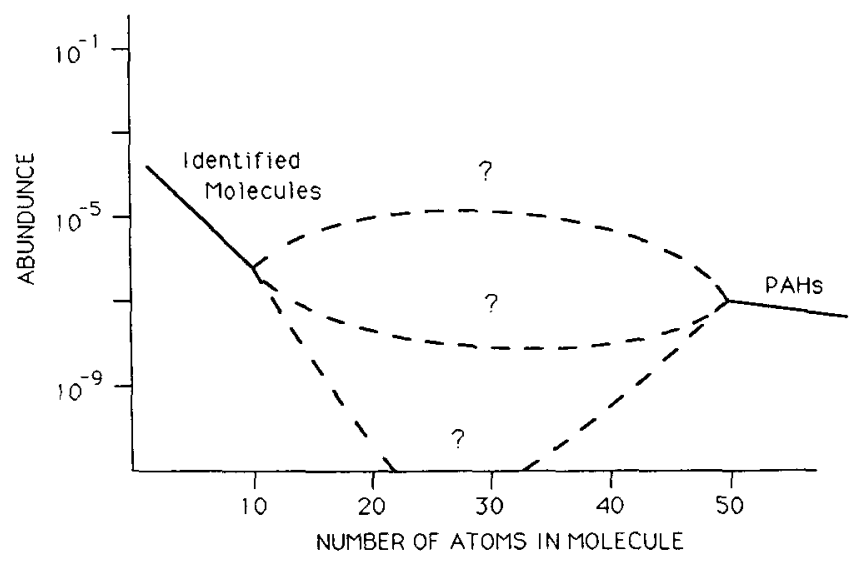

Fig. 7. Showing the gap in observed species in the 13 to 50 atom range.

feature of interstellar chemistry. Figure 7 shows the currently known abundances as a function of the number of atoms in a molecule.

SMMM will address this problem in two ways. First, if there is a continuum of heavy molecules, up to the 50 atom range of the PAHs, SMMM will be able to observe them by means of their vibration spectra. For example, the vibrationrotation bands of linear carbon chain molecules such as the well known interstellar cyano-polyacetylenes $\left(\mathrm{HC}_{3} \mathrm{~N}, \mathrm{HC}_{5} \mathrm{~N} \ldots \mathrm{HC}_{9} \mathrm{~N}\right)$ will fall in the $45-280 \mu \mathrm{m}$ range. The resolving power of the Fabry-Perot will be well suited to studies of the broad vibration-rotation bands. Second, SMMM will be able to study the PAHs themselves. The PAHs, which were identified by their strong resonances between 3-11 $\mu \mathrm{m}$, are known from theoretical and laboratory studies to have spectral features out to $100 \mu \mathrm{m}$ due to low-frequency lattice vibration modes. For example, the lowest frequency vibrational mode of coronene ( $24 \mathrm{C}$ atoms) is at about $100 \mu \mathrm{m}$. Comparable transitions for larger, heavier molecules will appear at longer wavelengths. These features may be quite strong since PAHs are expected to have permanent dipole moments either because they are ionized or because a peripheral hydrogen atom has been replaced by a radical. Finally, it is possible that other forms of dust particles, both with and without mantles, may possess spectral features in the submillimeter, as do many materials in the laboratory at low temperatures. Emission from both polar and non-polar molecules trapped in grain lattice vacancies can lead to observable features in the $70-150 \mu \mathrm{m}$ range.

\subsection{Galaxies and Cosmology}

The capability of the proposed mission for spectroscopic, high spatial-resolution imaging of molecular and fine-structure transitions and for high angular resolution continuum measurements will make maj or contributions to understanding the structure and evolution of galaxies. Just as for the interstellar medium of the Galaxy, the most important molecular transitions in external galaxies will be those of $\mathrm{CO}$ and of hydrides, such as $\mathrm{H}_{2} \mathrm{O}$. Over a broad range of physical conditions, the 


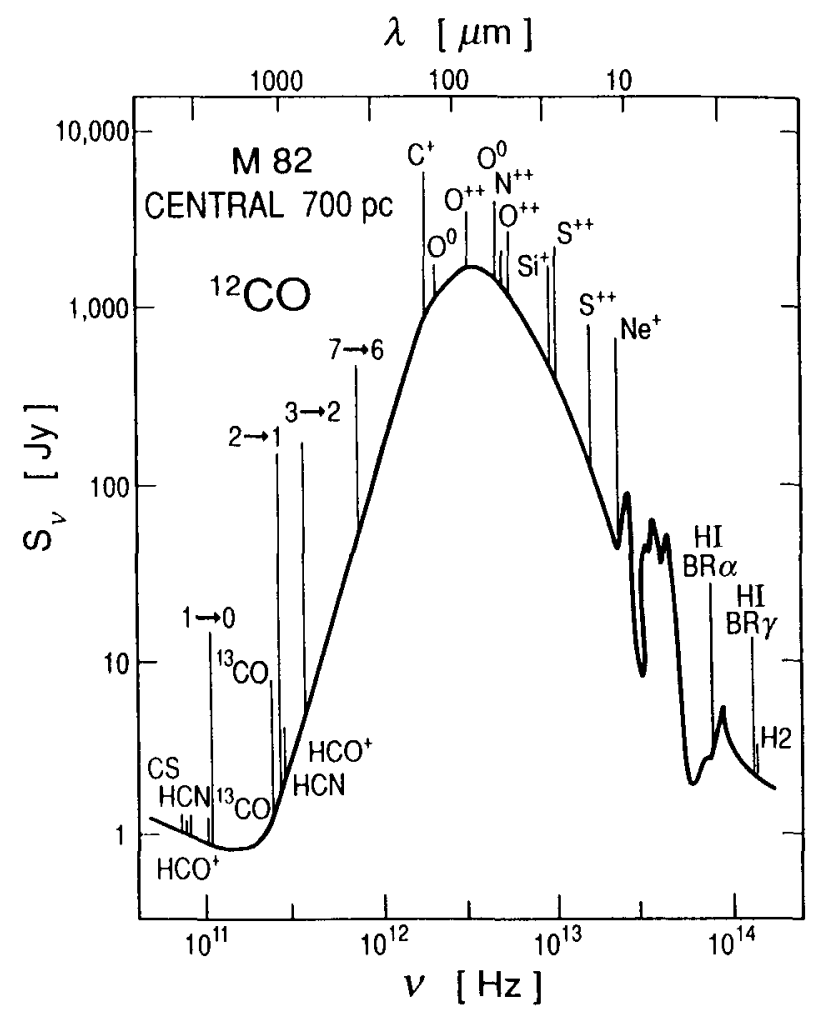

Fig. 8. Observed lines in the nearby starburst galaxy M82.

major cooling transitions for atomic gas are O I and C II. As for galactic clouds dust emission occurs in the submillimeter. These tracers may be observed with virtual immunity to the obscuration which plagues optical studies of galactic structure. Also, for nearby galaxies the opportunity will be present to study the variations of relative atomic abundances by observing the hydrides and comparing these with equivalent variations deduced from optical spectroscopy. Figure 8 shows a wide variety of lines already detected from the nearby galaxy M82. These will, of course, be supplemented by many of the lines from figure 3, particularly $\mathrm{H}_{2} \mathrm{O}$. Because of the freedom from atmospheric absorption these lines can be used, no matter what the red-shift of the galaxy.

\subsubsection{Star-formation in nearby galaxies}

The positions of atomic, molecular, and ionized gas regions relative to the galactic structures (e.g., the spiral arms and bars) are critical in understanding the largescale patterns of star-formation in galaxies. Important issues are whether starforming gas clouds are created within the spiral arms or exist prior to their entry into the arms and how star-formation is initiated in these clouds. In the past, it 
has been assumed that the molecular clouds are formed within the spiral arms, as low-density atomic gas is compressed by the spiral density-wave. Once formed, the molecular clouds would readily undergo massive star-formation. On the other hand, recent millimeter observations of the molecular gas in nearby external galaxies have clearly shown that molecular clouds exist in the inter-arm regions and, thus, the gas which enters the arms is already in the form of dense molecular clouds. The formation of massive stars selectively in the spiral arm clouds must then be initiated by phenomena unrelated to cloud formation, possibly cloud-cloud collisions, which become more frequent when cloud orbits crowd in the arms. In this picture, the formation of massive stars will be preceded by an enhancement in the number density of clouds and the emission of shock-excited molecular transitions occurring at the interface of colliding clouds. One of the primary signatures of shocked, high density, high temperature regions is $\mathrm{H}_{2} \mathrm{O}$ line emission. Mapping selected regions for emission from shocked, high-excitation molecular gas $\left(\mathrm{H}_{2} \mathrm{O}\right.$ and $\left.\mathrm{OH}\right)$ and ionized gas (C II and N II) will permit elucidation of the temporal sequence of cloud evolution and star-formation relative to the spiral arms. To map an entire galaxy of 0.1 square degrees, in a ground-state water line, would take about four hours with SMMM.

\subsubsection{IRAS Galaxies.}

One of the most spectacular results of the IRAS survey was the discovery of a class of extremely luminous galaxies, typified by Arp 220 and NGC 6240, which emit over $90 \%$ of their energy at far-infrared wavelengths. The survey has located about 25,000 galaxies, about half of which were previously uncataloged. Many of these galaxies emit most of their total energy in the submillimeter; many have luminosities in excess of $10^{11} \mathrm{~L}_{\odot}$, and some have luminosities exceeding $10^{12} \mathrm{~L}_{\odot}$. These large luminosities are typical of quasars and BL Lac objects and, thus, the IRAS galaxies form a bridge in luminosity between normal galaxies and the galaxies with active nuclei. Elucidating the origin of the activity in these galaxies will be a vital step towards understanding distant quasars. These galaxies are a significant constituent of the universe. Above $2 \times 10^{11} \mathrm{~L}_{\odot}$, the space density of IRAS galaxies exceeds that of normal galaxies, above $\sim 10^{12} \mathrm{~L}_{\odot}$, it exceeds that of the quasars. The IRAS galaxies must be studied at submillimeter wavelengths in order to answer the basic questions relating to their origin and luminosity source. IRAS has vividly demonstrated that interactions can play a dominant role in the evolution of galaxies, possibly leading to the formation of elliptical galaxies, and perhaps even leading to the formation of quasars via the funneling of gas to the nuclei of galaxies and the subsequent evolution of this gas as it falls into a massive black hole. How the interaction of gas-rich galaxies propagates is a problem that must be solved observationally. Because the interactions trigger outbursts of star-formation in dusty regions, the line and continuum mapping capabilities of SMMM are ideal in tracing the locations of gas concentrations, shocked gas, and embedded luminosity sources in galaxies undergoing the full range of interactions from initial contact through to completed merger.

At this time, only a few of the brightest IRAS galaxies have been measured at 
wavelengths longer than $100 \mu \mathrm{m}$. The sensitivity of SMMM (30 mJy, $1 \sigma$ in 1 second, in the continuum at $100 \mu \mathrm{m}$ ) is well matched to the faintest galaxies measured by IRAS ( $200 \mathrm{mJy}$ at $60 \mu \mathrm{m}$ ), so the bolometers on the proposed SMMM should be able to complete the determination of the full continuum energy distribution of large numbers of galaxies.

\subsubsection{Cosmology and the Evolution of High $z$ Galaxies.}

Among the central issues in observational cosmology today are the formation of galaxies and the large-scale structure (LSS), and the origins of non-thermal nuclear activity in quasars and other active galaxies. Both issues are inseparable from understanding galaxy evolution at large red-shifts. The former also includes the questions of the initial chemical enrichment, and the nature of the dark matter. Studies at submillimeter wavelengths can provide valuable clues, and perhaps even solutions for some of these important problems.

There is a large gap in our empirical knowledge about the universe between the epoch corresponding to the most distant quasars known, at $z \sim 4$, and the epoch of decoupling, the cosmic microwave background (CMBR) photosphere, at $\mathrm{z}$ $\sim 10^{3}$. Many cosmologically important processes are likely to have happened in that interval, e.g., the early stages, or perhaps even the bulk of galaxy formation and the initial chemical enrichment, development of the LSS, the appearance of quasars, etc. Galaxies are now known out to $\mathrm{z} \sim 3$ to 4 and clusters of galaxies out to $\mathrm{z} \sim 1$. The density contrast of the LSS today is about a factor of two, with rich clusters and galaxies representing much higher peaks, whereas the CMBR photosphere is smooth to about one part in $10^{5}$, or even less, on angular scales corresponding to galaxies and the LSS today. This density contrast must have developed, and the accompanying release of binding and other energy must have happened, in the red-shift interval $z \sim 1$ to $10^{3}$, possibly with the addition of some exotic or highly energetic phenomena. The generic expectation is that the energy released in these epochs would now be observable in the submillimeter region, due to the red-shifting, and possibly also due to reprocessing by dust at large red-shifts.

Hubble Space Telescope will provide much more information on the formative and evolutionary processes of distant galaxies than we have today, but if the initial processes include the generation of dust, the most interesting situations will be hidden to it. The information from the submillimeter band will then be not just complementary, but critical.

The extreme sensitivity of SMMM implies the ability to probe galaxies with large red-shifts. As shown in Figure 9, star-burst galaxies of $10^{12} \mathrm{~L}_{\odot}$ and with an energy distribution similar to M82 will be detectable in the continuum ( $6 \sigma$ in one hour) up to a red-shift of 2.5. Using the heterodyne receivers, the sensitivity for lines is $2 \times 10^{-18} \mathrm{~W} / \mathrm{m}^{2}$ ( $6 \sigma$ in one hour). Assuming that $5 \times 10^{-3}$ of the total luminosity is in the C II line at $158 \mu \mathrm{m}$, such galaxies can be observed in this line up to a red-shift of $z=2$ for $10^{12} \mathrm{~L}_{\odot}$ galaxies. The high spectral resolution of the heterodyne receivers will provide detailed information on the dynamics of these active objects. There is no other method to obtain this information. The C II line does not enter the heterodyne band until $z \geq 1$, so for lower red-shifts the Fabry- 


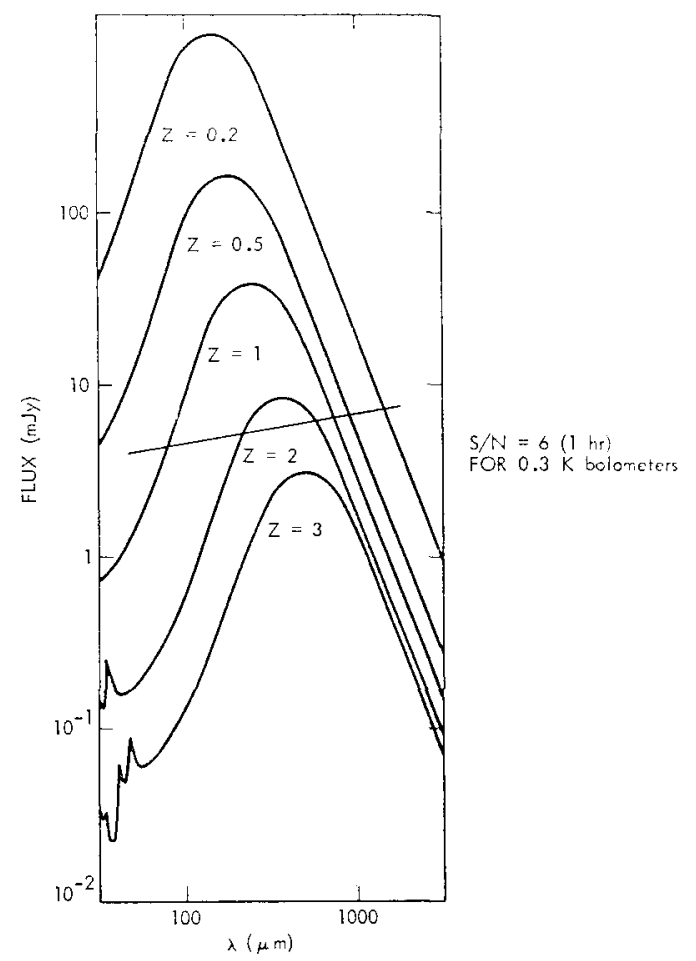

Fig. 9. Plots of the flux from a star-burst galaxy with a spectrum similar to M82, as a function of $z$. The galaxy is assumed to have an intrinsic luminosity of $10^{12} \mathrm{~L}_{\odot}$. Detections will be possible to $z \approx 2.5$, as shown by the line representing the SMMM sensitivity limit for a signal-to-noise ratio of 6 after one hour of integration with $0.3 \mathrm{~K}$ bolometers. The line spectra of Figure 8 scale with the continuum shown here.

Perot is used. Again, due to the very high sensitivity of the bolometers, detections of C II will be made in only minutes.

A principal use of the very sensitive continuum measurements will be to search for evolutionary effects in galaxies. The present, best luminosity function of the IRAS galaxies in the local universe is based upon the IRAS bright galaxy sample with $f_{\nu}(60 \mu \mathrm{m}) \geq 5 \mathrm{Jy}$. The mean red-shift of the galaxies in the bright galaxy sample is $z \sim 0.006$ and red-shifts extend to $z=0.08$. Although the ultra-deep surveys made with the IRAS satellite go as faint as $100 \mathrm{mJy}$ at $100 \mu \mathrm{m}$, these are severely confusion limited, and the real potential of SMMM in studying high redshift galaxies will be realized only at larger red-shifts than those found by IRAS. With the bolometer array, a survey for very high red-shift galaxies is feasible. In 300 hours of observing time, approximately one square degree of sky could be searched to a sensitivity of about $10 \mathrm{mJy}(1 \sigma=3 \mathrm{mJy})$ in three wavelength bands. If there were no evolution, about 500 galaxies should be detected to this limit within this area, and the sample would have a mean red-shift of $\mathrm{z} \sim 0.15$ and would contain some objects with red-shifts $\sim 2$. The areas chosen for such a survey would have to 
be carefully selected to avoid galactic "infrared cirrus" and known galaxy clusters. Because of confusion due to multiple sources in the field, SMMM will have very superior sensitivity as compared to the cooled, but smaller telescopes ISO and SIRTF, for wavelengths greater than $200 \mu \mathrm{m}$.

An important capability, provided by the long wavelength continuum camera, will be the study of perturbations to the microwave background. Although we are currently waiting for COBE results to define the future areas for study, it is clear that SMMM will be able to make definitive images of any submillimeter spectral distortions such as that detected at $600 \mu \mathrm{m}$ in the Nagoya-Berkeley experiment. A second distortion which could be measured is that caused by the Sunyaev-Zel'dovich effect. By making measurements at several different wavelengths SMMM would be able to separate the effects of temperature, radial velocity with respect to the CBR and opacity of the cluster gas. The measurement of the peculiar velocities of galaxy clusters could be made with a precision of about $150 \mathrm{~km} / \mathrm{sec}$, independent of the absolute magnitude of the red-shift itself. 